Article

\title{
Commutative Topological Semigroups Embedded into Topological Abelian Groups
}

\author{
Julio César Hernández Arzusa
}

Programa de Matemáticas, Universidad de Cartagena, Campus San Pablo, Cartagena CP 130014, Colombia; jhernandeza2@unicartagena.edu.co

Received: 19 June 2020; Accepted: 20 July 2020; Published: 24 July 2020

check for updates

\begin{abstract}
In this paper, we give conditions under which a commutative topological semigroup can be embedded algebraically and topologically into a compact topological Abelian group. We prove that every feebly compact regular first countable cancellative commutative topological semigroup with open shifts is a topological group, as well as every connected locally compact Hausdorff cancellative commutative topological monoid with open shifts. Finally, we use these results to give sufficient conditions on a commutative topological semigroup that guarantee it to have countable cellularity.
\end{abstract}

Keywords: cancellative topological semigroup; cellularity; $\sigma$-compact space; feebly compact space

MSC: Primary: 54B30, 18B30, 54D10; Secondary: 54H10, 22A30

\section{Introduction}

In this paper, we shall study the embedding of a commutative topological semigroup into a topological Abelian group as an open subsemigroup. We take advantage of these results to find conditions under which a cancellative commutative topological semigroup has countable cellularity, as well as when a topological semigroup is a topological group.

The main results are in Section 3, which we start with algebraic commutative semigroups embedded in groups. This topic is part of time-honored problem: When can such a semigroup be considered as a subsemigroup of a group? For algebraic semigroups it is known that a commutative semigroup can be embedded in a group if and only if it is cancellative, but in non-commutative semigroups the cancellation is not a sufficient condition. Ore's Theorem gives us a sufficient condition for embeddability in a group (see [1] (Section 1.10)) (Section 1.10). Because the context in the Section 3 is topological embedding of topological semigroups in topological groups, additional related topics arise. In [2], Neal Rothman presents some properties that allow for us to embed (topologically) a cancellative commutative topological semigroup into a topological group. For example, he proved that every commutative cancellative Hausdorff topological semigroup with open shifts and continuous division can be embedded into a topological group as a open subsemigroup [2] (Theorem 1.19). We prove that a commutative cancellative Hausdorff locally compact topological semigroup with open shifts has continuous division and, therefore, it can be embedded into topological group (Theorem 1), if in addition the semigroup is also pseudocompact, then it can be embedded into compact topological group as a dense open subsemigroup (Theorem 2). This result can be viewed as an extension of [3] (Theorem 2.3.2). We finalized the Section 3 with Compactness type conditions under which a topological semigroup is a topological group (Theorems 3 and 4). Similar results are studied since the 50ies of the last century. For example, as an exercise on [4] (p. 77) we find that the closure of the a subsemigroup of a compact group is a topological group. This last result was established in [5] and it was extended to countably compact first countable Hausdorff topological semigroups in [6], but [7] establishes that the first countableness condition cannot be removed. Bokalo, B. and Guran, I. 
[8] (Theorem 6) tell us that any sequentially compact Hausdorff cancellative topological semigroup is a topological group. However it is still unknown if (in ZFC) any cancellative countably compact regular topological semigroup is a topological group (see [9] (Problem 2.5.1)), this question was raised by A. D. Wallace in 1955. Accordingly, the idea that a cancellative topological semigroup, with some compactness type conditions, is a group is likely to be some seventy years old. For our results, we used feeble compactness, local compactness, and pseudocompactness.

In Section 4, we present sufficient conditions under which a topological semigroup have countable cellularity, the reflection on the class of regular spaces allows for us to disregard the axioms of separation to obtain topological monoids with countable cellularity. The class of topological spaces having countable cellularity is wide, in fact, it contains (among other classes) the class of the $\sigma$-compact paratopological groups (see [10] (Corollary 2.3)), the class of sequentially compact $\sigma$-compact cancellative topological monoids (see [11] (Theorem 4.8)) and the class of subsemigroups of precompact topological groups (see [12] (Corollary 3.6)). However there are still open problems that are related to this topic, for example, it is unknown if a countably compact Hausdorff topological semigroup has countable cellularity (see [9] (Problem 2.5.2)).

\section{Preliminaries}

We denote by $\mathbb{Z}, \mathbb{R}$ and $\mathbb{N}$, the set of all the integer numbers, real numbers, and positive integer numbers, respectively. If $A$ is a set, $|A|$ will denote the cardinal of $A, \aleph_{0}=|\mathbb{N}|$. If $X$ is a topological space and $x \in X, N_{x}^{(X)}$ will denote the set of all open neighborhoods of $x$ in $X$ or simply $N_{x}$ when the space is understood.

A semigroup is a set $S \neq \varnothing$, endowed with an associative operation. If $S$ also has neutral element, we say that $S$ is a monoid. A mapping $f: S \longrightarrow H$ between semigroups is a homomorphism if $f(x y)=f(x) f(y)$ for all $x, y \in S$. A semitopological semigroup (monoid) consists of a semigroup (resp. monoid) $S$ and a topology $\tau$ on $S$, such that, for all $a \in S$, the shifts $x \mapsto a x$ and $x \mapsto x a$ (noted by $l_{a}$ and $r_{a}$, respectively) are continuous mappings from $S$ to itself. We say that a semitopological semigroup has open shifts, if for each $a \in S$ and for each open set $U$ in $S$, we have that $l_{a}(U)$ and $r_{a}(U)$ are open sets in S. A topological semigroup (monoid) (paratopological group) consists of a semigroup (resp. monoid) (resp. group) $S$ and a topology $\tau$ on $S$, such that the operation of $S$ is jointly continuous. Like [9], we do not require that semigroups to be Hausdorff. If $S$ is a paratopological group and if also the mapping $x \mapsto x^{-1}$ is continuous, we say that $S$ is a topological group. A congruence on a semigroup $S$ is an equivalence relation on $S, \sim$, such that if $x \sim y$ and $a \sim b$, then $x a \sim y b$. If $S$ is a semitopological semigroup, then we say that $\sim$ is a closed congruence if $\sim$ is closed in $S \times S$. If $\sim$ is an equivalence relation in a semigroup (monoid) $S$ and $\pi: S \longrightarrow S / \sim$ is the respective quotient mapping, then $S / \sim$ is a semigroup (monoid) and $\pi$ a homomorphism if and only if $\sim$ is a congruence ([13], Theorem 1).

The axioms of separation $T_{0}, T_{1}, T_{2}, T_{3}$, and regular $\left(T_{r}\right)$ are defined in accordance with [14]. We denote by $\mathcal{C}_{i}$ the class of the $T_{i}$ spaces, where $i \in\{0,1,2,3, r\}$.

If $X$ is a topological space and $A \subseteq X$, we will note by $\operatorname{Int}_{X}(A)$ and $C l_{X}(A)$, the interior and the closure of $A$ in $X$, or simply $\operatorname{Int}(A)$ and $\bar{A}$, respectively, when the space $X$ is understood. An open set $U$ in $X$, is called regular open in $X$ if $\operatorname{Int} \bar{U}=U$. It is easy to prove the regular open ones form a base for a topology in $X, X$ endowed with this topology, will note by $X_{s r}$, which we will call semiregularization of $X$.

A space $X$ is locally compact if each $x \in X$ has a compact neighborhood.

\section{Embedding Topological Semigroups into Topological Groups}

Let $S$ be a cancellative commutative semigroup, $S \times S$ is a cancellative commutative semigroup, by defining the operation coordinatewise. Let us define in $S \times S$ the following relation: $(x, y) \mathcal{R}(a, b)$ if and only if $x b=y a$. It is not to hard to prove that $\mathcal{R}$ is a congruence, hence if $\pi: S \times S \longrightarrow(S \times S) / \mathcal{R}$ is the respective quotient mapping, the operation induced by $\pi$ makes of $(S \times S) / \mathcal{R}$ a semigrup. It 
is easy to prove that $(S \times S) / \mathcal{R}$ is a group, where the equivalence class $\{\pi((x, x)): x \in S\}$ is the neutral element, and the inverse of $\pi((a, b))$ is $\pi((b, a))$. Additionally, the function $\iota: S \longrightarrow(S \times S) / \mathcal{R}$ defined by $\iota(x)=\pi((x a, a))$, for each $x \in S$, is an algebraic monomorphism, where $a$ is a fixed element of $S(\iota$ does not depend of the choice of $a)$. Note that $\pi(x, y)=\pi\left(x a^{2}, y a^{2}\right)=\pi(x a, a) \pi(a, y a)=$ $\iota(x)(\iota(y))^{-1}$, therefore if we identify each element of $S$ with its image under $\iota$, we have that each element of $(S \times S) / \mathcal{R}$ can be written as $x y^{-1}$, where $x, y \in S$, that is to say $(S \times S) / \mathcal{R}=S S^{-1}$. Let $f: S \longrightarrow G$ be a homomorphism to an abelian topological group $G$, there is an unique homomorphism $f^{*}:(S \times S) / \mathcal{R} \longrightarrow G$ (defined as $f^{*}\left(\pi((x, y))=x y^{-1}\right)$ such that $\left.f^{*}\right|_{S}=f$. In summary, we have that the class of the abelian groups is a reflective subcategory of the class of the cancellative commutative semigroups. Because the reflections are unique up to isomorphims, $(S \times S) / \mathcal{R}$ is uniquely determined by $S$, and we will denote by $\langle S\rangle$ and it is called group generated by $S$. If $S$ is a topological semigroup, we will call $\langle S\rangle^{*}$ to $\langle S\rangle$ endowed with the quotient topology induced by $\pi: S \times S \longrightarrow\langle S\rangle$, where $S \times S$ is endowed with the Tychonoff product topology.

Definition 1. Let $S$ be a topological semigroup. We say that $S$ has continuous division if give $x, y \in S$ and an open set $V$ in $S$, containing $y$, there are open sets in $S, U$, and $W$, containing $x$ and $x y(y x)$, respectively; such that $W \subseteq \bigcap\{u V: u \in U\}(W \subseteq \bigcap\{V u: u \in U\})$.

Proposition 1 ([2] (Theorem 1.15 and Theorem 1.19)). If $S$ is a cancellative and commutative Hausdorff topological semigroup with open shifts, then $\langle S\rangle^{*}$ is a Hausdorff topological group and the quotient mapping $\pi: S \times S \longrightarrow\langle S\rangle^{*}$ is open. Furthermore, if $S$ has continuous division, $\iota: S \longrightarrow \iota(S)$ is a homeomorphism and $\iota(S)$ is open in $\langle S\rangle^{*}$.

Proposition 2. Every open subsemigroup of a topological group has continuous division.

Proof. Let $S$ be an open subsemigroup of a topological group $G$. Let $x, y \in S$, since $G$ is a group, $x^{-1} x y=y$. Let $V$ be an open subset of $S$ containing $y$, then $V$ is open in $G$, the continuity of the operations of $G$, implies that there are open subsets in $G, K$ and $M$, containing $x$ and $x y$, respectively, such that $K^{-1} M \subseteq V$. Let us put $U=K \cap S$ and $W=M \cap S$, then $U$ and $W$ are open subsets of $S$ containing $x$ and $x y$, respectively. Now, if $t \in W$ and $u \in U$, then $u^{-1} t \in K^{-1} M \subseteq V$, hence $t \in u V$, therefore $W \subseteq u V$, for every $u \in U$. We have proved that $W \subseteq \bigcap_{u \in U} u V$. For $y x$, we proceed analogously.

So far, we just have embedded, algebraically, semigroups into groups, the following proposition gives us a topological and algebraic embedding.

Proposition 3. Let $S$ be a cancellative commutative semitopological semigroup with open shifts. There exists a topology $\tau$ in $\langle S\rangle$, such that $(\langle S\rangle, \tau)$ is a semitopological group containing $S$ as an open semigroup. Moreover

(i) $S$ is first countable if and only if $(\langle S\rangle, \tau)$ is first countable.

(ii) $(\langle S\rangle, \tau)$ is a paratopological group if and only if $S$ is a topological semigroup.

(iii) If $S$ is $T_{2},(\langle S\rangle, \tau)$ is $T_{2}$.

(iv) If $S$ is Hausdorff and locally compact, $(\langle S\rangle, \tau)$ is a locally compact Hausdorff topological group.

Proof. Let $x$ be a fixed element in $S$, where $S$ is a cancellative commutative semitopological semigroup with open shifts, and put $\mathcal{B}=\left\{x^{-1} V: V \in N_{x}^{(S)}\right\}$. We will prove that $\{g U: U \in \mathcal{B}, g \in\langle S\rangle\}$ is a base for a topology making $\langle S\rangle$ into semitopological group, for it we will prove the conditions 1,3 , an 4 given in [15] (p. 93). It is easy to prove the conditions 1 and 4 , let us see 3. Let $V_{x} \in N_{x}^{(S)}$ and let $t \in x^{-1} V_{x}$. From the fact that $\langle S\rangle=S S^{-1}$, we have that $t=a b^{-1}$, where $a, b \in S$, thus $a x \in b V_{x}$. Given that the shifts in $S$ are open and continuous, we can find $W_{x} \in N_{x}^{(S)}$, such that $a W_{x} \subseteq b V_{x}$, then $t\left(x^{-1} W_{x}\right) \subseteq x^{-1} V_{x}$ and condition 3 holds. If $\tau$ is the topology generated by $\mathcal{B}$, then $(\langle S\rangle, \tau)$ is a semitopological group; moreover, since $S \in N_{x}, S=x\left(x^{-1} S\right)$, we have that $S$ is open in $(\langle S\rangle, \tau)$. 
We will prove that $\left\{g x^{-1} V: V \in N_{x}^{(S)}\right\}$ is a local base at $g$, for every $g \in\langle S\rangle$. Indeed, let $U$ be an open set in $(\langle S\rangle, \tau)$ and $g \in U$, then there is $y \in\langle S\rangle$, such that $g \in y x^{-1} V \subseteq U$. There are $a, b, s, t \in S$, such that $y=a b^{-1}$ and $g=t s^{-1}$; therefore, $t s^{-1} \in a b^{-1} x^{-1} V_{x} \subseteq U$, so that $t b x \in a s V_{x} \subseteq b x s U$. Hence, there is $W_{x}$ open in $S$ satisfying $t b W_{x} \subseteq a s V_{x} \subseteq b x s U$; therefore, $g\left(x^{-1} W_{x}\right) \subseteq U$, this implies that $\left\{g x^{-1} V: V \in N_{x}^{S}\right\}$ is a local base at $g$.

Let us see that $S$ is a subspace of $(\langle S\rangle, \tau)$. Indeed, let $U$ be an open set in $S$ and let $s \in U$. Subsequently, $x s \in x U$, and so there is a $V \in N_{x}^{(S)}$ such that $s V \subseteq x U$. This is equivalent to saying that $s\left(x^{-1} V\right) \subseteq U$, so that $s \in\left(s\left(x^{-1} V\right)\right) \cap S \subseteq U$, therefore $U$ is open in the topology of subspace of S. Reciprocally, let $U$ be an open set in $(\langle S\rangle, \tau)$ and $s \in U \cap S$, we can find $W_{x} \in N_{x}^{S}$ and $U_{s} \in N_{s}^{(S)}$, such that $s \in s x^{-1} W_{x} \subseteq U, x U_{s} \subseteq s W_{x} \subseteq x U$, then $U_{s} \subseteq U$ and, therefore, $U_{s} \subseteq U \cap S$, this proves that $U \cap S$ is open in $S$. From the fact that $\left\{g x^{-1} V: V \in N_{x}^{(S)}\right\}$ is a local base at $g$, it follows that if $S$ is first countable (locally compact), then $(\langle S\rangle, \tau)$ is also first countable (resp. locally compact).

Let us suppose that $S$ is a topological semigroup and let us prove that $(\langle S\rangle, \tau)$ is a paratopological group, it can be concluded if we prove that the condition 2 of [15] (Page 93) holds for $\mathcal{B}$. Indeed, let $V_{x} \in N_{x}^{(S)}$, since $x^{2} \in x V_{x}$ and the operation in $S$ is jointly continuous, there exists $W_{x} \in N_{x}^{(S)}$, such that $\left(W_{x}\right)^{2} \subseteq x V_{x}$, then $\left(x^{-1} W_{x}\right)^{2} \subseteq V_{x}$ and condition 2 holds, this proves that $(\langle S\rangle, \tau)$ is a paratopological group. Let us suppose that $S$ es $T_{2}$ and let us see $(\langle S\rangle, \tau)$ is $T_{2}$, indeed let $y, z \in\langle S\rangle, z \neq y$, then there are $a, b, c, d \in S$, such that $z=a b^{-1}$ and $y=c d^{-1}$, so that $a d \neq b c$, by fact that $S$ is $T_{2}$, we can obtain $V_{a d} \in N_{a d}^{(S)}$ and $V_{b c} \in N_{b c}^{(S)}$. Note that $\left((b d)^{-1} V_{a d}\right) \cap\left((b d)^{-1} V_{b c}\right)=\varnothing$, also, $\left.(b d)^{-1} V_{a d}\right) \in N_{z}^{(\langle S\rangle)}$ and $\left.(b d)^{-1} V_{b c}\right) \in N_{y}^{(\langle S\rangle)}$, that is to say $(\langle S\rangle, \tau)$ es $T_{2}$. Finally, if $S$ is locally compact and $T_{2},(\langle S\rangle, \tau)$ is a semitopological group locally compact and $T_{2}$ by Ellis' Theorem $(\langle S\rangle, \tau)$ is a topological group.

From the item (ii) of the Propositions 2 and 3, we have the following result.

Corollary 1. If $S$ is a cancellative commutative locally compact Hausdorff semitopological semigroup with open shifts, then $S$ has continuous division.

The following theorem tells us that every cancellative commutative locally compact Hausdorff topological semigroup with open shifts can be embedded as an open semigroup into the locally compact Hausdorff topological group, $\langle S\rangle^{*}$.

Theorem 1. Let $S$ be a cancellative, commutative topological semigroup with open shifts. If $S$ is Hausdorff and locally compact, then so is $\langle S\rangle^{*}$. Moreover, $\iota: S \longrightarrow \iota(S)$ is a homeomorphism and $\iota(S)$ is open in $\langle S\rangle^{*}$.

Proof. Because $S$ is locally compact and Hausdorff topological semigroup, so is $S \times S$. By virtue, the Proposition 1, $\pi: S \times S \longrightarrow\langle S\rangle^{*}$ is open and $\langle S\rangle^{*}$ is Hausdorff, hence $\langle S\rangle^{*}$ is locally compact Hausdorff topological group. From Corollary 1 , it follows that $S$ has continuous division, therefore the Proposition 1 guarantees that $\iota: S \longrightarrow \iota(S)$ is a homeomorphism and $\iota(S)$ is open in $\langle S\rangle^{*}$.

From [3] (Theorem 2.3.2), it is known that every pseudocompact Tychonoff topological group, $S$, can be embedded as a subgroup dense into a compact topological group, the following theorem presents an analogous result in the context of cancellative commutative topological semigroups, where, in addition to pseudocompactness of $S$, its local compactness is also required.

Theorem 2. If $S$ is a cancellative commutative locally compact pseudocompact Hausdorff topological semigroups with open shifts, then $S$ is an open dense subsemigroup of $\langle S\rangle^{*}$ and $\langle S\rangle^{*}$ is a compact topological group.

Proof. Because $S$ is a locally compact pseudocompact space, [14] (Theorem 3.10.26) implies that $S \times S$ is pseudocompact. From the fact that $\langle S\rangle^{*}$ is a continuos image of $S \times S$, we have that $\langle S\rangle^{*}$ is pseudompact, therefore the Cěch-Stone compactification, $\beta\langle S\rangle^{*}$, is a topological group containing a $\langle S\rangle^{*}$ as dense subgroup. [14] (Theorem 3.3.9) guarantees that $\langle S\rangle^{*}$ is an open subgroup of $\beta S$, 
therefore it is also closed. By the density of $\langle S\rangle^{*}$, we have that $\langle S\rangle^{*}=\beta\langle S\rangle^{*}$, that it to say, $\langle S\rangle^{*}$ is a compact topological group. Because $\langle S\rangle^{*}$ is compact, $S$ is open in $\langle S\rangle^{*}$ and $\langle S\rangle^{*}=S S^{-1}$, there are $s_{1}, s_{2} \ldots, s_{n}$ in $S$, such that $\langle S\rangle^{*}=\bigcup_{i=1}^{n} S s_{i}^{-1} . C l_{\langle S\rangle^{*}}(S)$ is a compact Hausdorff cancellative semigroup, then by [9] (Theorem 2.5.2), $\mathrm{Cl}_{\langle S\rangle^{*}}(S)$ is a topological group, therefore $s_{i}^{-1} \in \mathrm{Cl}_{\langle S\rangle^{*}}(S)$ for every $i \in\{1,2,3 \ldots, n\}$, hence $\left(C l_{\langle S\rangle^{*}}(S)\right) s_{i}^{-1}=C l_{\langle S\rangle^{*}}(S)$ for every $i \in\{1,2,3 \ldots, n\}$. Because each shift in $\langle S\rangle^{*}$ is a homeomorphism, we have that $\langle S\rangle^{*}=C l_{\langle S\rangle^{*}}\left(\bigcup_{i=1}^{n} r_{s_{i}^{-1}}(S)\right)=\bigcup_{i=1}^{n} C l_{\langle S\rangle^{*}}\left(r_{s_{i}^{-1}}(S)\right)=$ $\bigcup_{i=1}^{n} r_{s_{i}^{-1}}\left(C l_{\langle S\rangle^{*}}(S)\right)=\bigcup_{i=1}^{n}\left(C l_{\langle S\rangle^{*}}(S)\right) s_{i}^{-1}=\bigcup_{i=1}^{n} C l_{\langle S\rangle^{*}}(S)=C l_{\langle S\rangle^{*}}(S)$, that it to say, $S$ is dense in $\langle S\rangle^{*}$.

We obtain the following corollary.

Corollary 2. The closure of any subsemigorup of a cancellative commutative locally compact pseudocompact Hausdorff topological semigroup with open shifts can be embedded as a dense open subsemigroup into a compact Hausdorff topological group.

Proof. Let $S$ be a cancellative commutative locally compact pseudocompact Hausdorff topological semigroup with open shifts and let $K$ be a subsemigorup of $S$. By Theorem $2, S$ is an open subsemigroup of $\langle S\rangle^{*}$, since $C l_{S}(K)=C l_{\langle S\rangle^{*}}(K) \cap S$, we have that $C l_{S}(K)$ is open in $C l_{\langle S\rangle^{*}}(K)$. Now, $K$ is dense in $C l_{\langle S\rangle^{*}}(K)$ and $K \subseteq C l_{S}(K) \subseteq C l_{\langle S\rangle^{*}}(K)$, this proves that $C l_{S}(K)$ is dense in $C l_{\langle S\rangle^{*}}(K)$, but $C l_{\langle S\rangle^{*}}(K)$ is a compact Hausdorff cancellative topological semigroup, so that it is a topological group by [9] (Theorem 2.5.2), and we have finished the proof.

Definition 2 ([16] (Section 2)). SAP-compactification of a semitopological semigroup $S$ is a pair $(G, f)$ consisting of a compact Hausdorff topological group $G$ and a continuous homomorphism $f: S \longrightarrow G$, such that for each continuous homomorphism $h: S \longrightarrow K$, where $K$ is a compact Hausdorff topological group, there is an unique continuous homomorphism $h^{*}: G \longrightarrow K$, such that $h=h^{*} \circ f$.

The following proposition gives us a realization of SAP-compactification (the notation came from the abbreviation for strongly almost periodic function rings that determine this compactification) in the class of cancellative commutative locally compact pseudocompact Hausdorff topological semigroups with open shifts.

Proposition 4. If $S$ is a cancellative commutative locally compact pseudocompact Hausdorff topological semigroup with open shifts, then $\left(\langle S\rangle^{*}, \iota\right)$ coincides with the SAP-compactification of $S$.

Proof. Let $S$ be a cancellative commutative locally compact pseudocompact Hausdorff topological semigroup with open shifts and $f: S \longrightarrow G$ a continuous homomorphism, being $G$ a Hausdorff compact topological group. Because $S$ is commutative, $f(S)$ is a commutative subsemigroup of $G$, so that $\overline{f(S)}$ is a compact commutative cancellative topological semigroup, which is a topological group by [9] (Theorem 2.5.2). Let us define $f^{*}:\langle S\rangle^{*} \longrightarrow \overline{f(S)}$ by $f^{*}\left(x y^{-1}\right)=f(x)(f(y))^{-1}, f^{*}$ is a continuous homomorphism and moreover $f^{*} \circ \iota=f$, this ends the proof.

We shall conclude this section by giving conditions under which a commutative topological semigroup is a topological group. We take advantage the reflection on the class of regular spaces. From [17], it is well known that for each $i \in\{0,1,2,3, r\} \mathcal{C}_{i}$ is a reflective subclass of the class of the topological spaces, it means given a space, $X$, there is a topological space, $\mathcal{C}_{i}(X) \in \mathcal{C}_{i}$, (unique up to homeomorphism) and a continuous mapping $\varphi_{\left(X, \mathcal{C}_{i}\right)}$ of $X$ onto $\mathcal{C}_{i}(X)$, such that given a continuous mapping $f: X \longrightarrow Y$, where $Y \in \mathcal{C}_{i}$, there exists an unique continuous mapping $g: \mathcal{C}_{i}(X) \longrightarrow Y$, such that $g \circ \varphi_{\left(X, \mathcal{C}_{i}\right)}=f$. The functor $\mathcal{C}_{i}$ is studied in $[10,18,19]$, in the category of semitopological groups. Similar results are found in $[11,20]$, but in the category of topological semigroups. The following proposition summarizes some properties in this regard. 
Proposition 5. Let S be a topological monoid with open shifts. Then

(i) $\mathcal{C}_{r}(S)$ is a monoid, $\varphi_{\left(S, \mathcal{C}_{i}\right)}$ is a homomorphism (see [20] (Proposition 3.8)) and $\mathcal{C}_{r}(S)=\mathcal{C}_{0}\left(S_{s r}\right.$ ) (see [11] (Theorem 2.8)). If $S$ is also cancellative, $\mathcal{C}_{r}(S)$ is cancellative (see [11] (Lemma 4.6)).

(ii) If $A$ is an open set in $S$, then $\mathcal{C}_{r}(A)=\varphi_{\left(S, \mathcal{C}_{r}\right)}(A)$ (see [20] (Corollary 5.7) and [21] (Lemma 4)).

(iii) $\mathcal{C}_{3}(S)=S_{s r}$. Moreover, if $S$ is $T_{2}, \mathcal{C}_{r}(S)=S_{s r}$ (see [11] (Corollary 2.7) and [21] (Proposition 1)).

(iv) $\varphi_{\left(S, \mathcal{C}_{i}\right)}$ is open for each $i \in\{0,1,2\}$ (see [11] (Proposition 2.1)).

(v) If $S$ is a paratopolgical group, $\mathcal{C}_{r}(S)$ is a paratopological group (see [19] (Corollary 3.3 and Theorem 3.8), [18] (Theorem 2.4)).

It is easy to see that if $X$ is a first countable topological space, then $X_{s r}$ is first countable. Since $\mathcal{C}_{r}(S)=\mathcal{C}_{0}\left(S_{S r}\right)$ and $\varphi_{\left(S, \mathcal{C}_{0}\right)}$ is open, whenever $S$ is a topological monoid with open shifts, we have the following corollary.

Corollary 3. If $S$ is a first countable topological monoid with open shifts, then $\mathcal{C}_{r}(S)$ is first countable.

Definition 3. A space $X$ is feebly compact if each locally finite family of open sets in $X$ is finite.

It is known that each pseudocompact Tychonoff paratopological group is a topological group (see [22] (Theorem 2.6)). The following theorem gives us a similar result in cancellative commutative topological monoids with open shifts, but instead of group structure we have required the first axiom of countability.

Theorem 3. Let $S$ be a cancellative commutative feebly compact topological monoid with open shifts satisfying the first axiom of countability. Subsequently, $\mathcal{C}_{r}(S)$ is a compact metrizable topological group. Moreover, the following statements hold:

(i) If $S$ is $T_{2}, S$ is a paratopological group.

(ii) If $S$ is regular, $S$ is a compact metrizable topological group.

Proof. Let $S$ be a commutative cancellative topological monoid with open shifts and put $G=\langle S\rangle$. From Proposition 3, we have that there is a topology $\tau$, such that $(G, \tau)$ is a paratopological group containing $S$ as an open monoid. It follows from Proposition 5 that $\mathcal{C}_{r}(G)$ is a regular paratopological group containing $\mathcal{C}_{r}(S)$, Accordingly, [23] (Corollary 5) implies that $\mathcal{C}_{r}(G)$ is Tychonoff, therefore so is $\mathcal{C}_{r}(S)$. Because $\mathcal{C}_{r}(S)$ is feebly compact and Tychonoff, by [3] (Theorem 1.1.3) it is pseudocompact. Afterwards, $\mathcal{C}_{r}(S)$ is a pseudocompact subspace of the regular first countable paratopological group $\mathcal{C}_{r}(G)$, following [24] (Corollary 4.18), we have that $\mathcal{C}_{r}(S)$ is metrizable and compact. By Proposition 5 (i), $\mathcal{C}_{r}(S)$ is cancellative, therefore [9] (Theorem 2.5.2) implies that $\mathcal{C}_{r}(S)$ is a topological group. Now, if $S$ is $T_{2}, \mathcal{C}_{r}(S)=S_{s r}$, but $S$ and $S_{s r}$ coincide algebraically, thus $S$ is a paratopological group. If $S$ is regular, then $S=\mathcal{C}_{r}(S)$, this ends the proof.

From [6], it is known that any cancellative first countable countably compact Hausdorff topological semigroup is a topological group. Since, in the class of Hausdorff spaces, countable compactness implies feeble compactness (see [14] (Theorem 3.10.2)), then Theorem 3 extends this result to the class of feebly compact semigroups.

By [3] (Example 2.7.10), there is a feebly compact Hausdorff second countable paratopological group that fails to be a compact topological group; therefore, the regularity in (ii) Theorem 3 cannot be weakened to the Hausdorff separation property.

Example 1. Let $\omega_{1}$ be the first non countable ordinal, the space $\left[0, \omega_{1}\right)$ of ordinal numbers strictly less than $\omega_{1}$ with its order topology is regular first-countable feebly compact space, but $\left[0, \omega_{1}\right)$ is not compact. Then we can see the importance of algebraic structure in the Theorem 3. 
Theorem 4. Every cancellative commutative locally compact connected Hausdorff topological monoid with open shifts is a topological group.

Proof. Let $S$ be a cancellative commutative locally compact connected Hausdorff and let $\tau$ be the topology given in the Proposition 3. Let $U \in N_{e_{S}}^{(S)}$, since $e_{S}=e_{\langle S\rangle}$ and $(\langle S\rangle, \tau)$ is a topological group, there is $V \in N_{e S}^{(\langle S\rangle)}$ satisfying $V^{-1}=V$ and $V \subseteq U$, then $\bigcup_{n \in \mathbb{N}} V^{n} \subseteq \bigcup_{n \in \mathbb{N}} U^{n}$. But $\bigcup_{n \in \mathbb{N}} V^{n}$ is an open subgroup of $(\langle S\rangle, \tau)$ and, therefore, is closed in $S$, the connectedness of $S$ implies that $\bigcup_{n \in \mathbb{N}} V^{n}=S$ and $S$ is a topological group.

\section{Cellularity of Topological Semigroups}

Finally, we present some results regarding the cellularity of topological semigroups.

Let $X$ be a topological space, a cellular family in $X$ is a pairwise disjoint non empty family of non empty open sets in $X$. The cellularity of a space $X$ is noted by $c(X)$ and it is defined by

$$
c(X)=\sup \{|U|: U \text { is cellular familiy in } X\}+\aleph_{0} .
$$

If $c(X)=\aleph_{0}$, we say that $X$ has countable cellularity or $X$ has the Souslin property.

Proposition 6 ([11] (Corolario 4.4)). If $S$ is a topological semigroup with open shifts, then $c(S)=c\left(\mathcal{C}_{r}(S)\right)$.

Theorem 5. Let $S$ be a cancellative, commutative Hausdorff locally compact $\sigma$-compact topological semigroup with open shifts. subsequently, $S$ has countable cellularity.

Proof. Because $S \times S$ is locally compact and $\sigma$-compact, from Proposition 1 we have that $\langle S\rangle^{*}$ is locally compact, Hausdorff, $\sigma$-compact topological group, which has countable celluarity following [10] (Corollary 2.3). Given that $S$ is open in $\langle S\rangle^{*}$, then $c(S)=c\left(\langle S\rangle^{*}\right)=\aleph_{0}$.

In the next corollary, we give an analogous result of the Proposition 5, but without considering axioms of separation.

Corollary 4. Every $\sigma$-compact locally compact cancellative commutative topological monoid with open shifts has countable cellularity.

Proof. Let $S$ be a $\sigma$-compact locally compact cancellative commutative topological monoid with open shifts. By Proposition $5 \varphi_{\left(S, \mathcal{C}_{2}\right)}$ is open, therefore $\mathcal{C}_{2}(S)$ is locally compact Hausdorff topological semigroup with open shifts, this implies that $\mathcal{C}_{2}(S)$ is regular, so that $\mathcal{C}_{2}(S)=\mathcal{C}_{r}(S)$. Because $\mathcal{C}_{r}(S)$ is cancellative, we can apply the Theorem 5 and the Proposition 6 to conclude that $c(S)=c\left(\mathcal{C}_{r}(S)\right)=\aleph_{0}$.

Ref. [10] (Corollary 2.3) tells us that $\sigma$-compact paratopological groups have countable cellularity, from this point of view, Theorem 5 and Corollary 4 are a generalization of this result to the category of topological semigroups, where in addition we have also required local compactness.

Corollary 5. Every subsemigroup of a commutative cancellative locally compact pseudocompact Hausdorff topological semigroup with open shifts has countable cellularity.

Proof. Let $S$ be a commutative cancellative locally compact pseudocompact Hausdorff topological semigroup with open shifts and let $K$ be a subsemigroup of $S$. By Corollary 2, there exists a compact Hausdorff topological group, $G$, containing $\mathrm{Cl}_{S}(K)$ as an open semitopological group, therefore $c(K) \leq c\left(C l_{S}(K)\right) \leq c(G)=\aleph_{0}$ 
Ref. [12] (Corollary 3.6) establishes that each subsemigroup of a precompact topological group has countable cellularity. Because pseudocompactness implies precompactness, then Corollary 5 extends [12] (Corollary 3.6) to the category of topological semigroups.

Since compact topological groups has countable cellularity and $c(S)=c\left(\mathcal{C}_{r}(S)\right)$ for every topological monoid with open shifts, then Theorem 3 implies the following corollary.

Corollary 6. Let $S$ be a cancellative commutative feebly compact topological monoid with open shifts satisfying the first axiom of countability. Subsequently, $S$ has countable cellularity.

Funding: This research received no external funding.

Acknowledgments: The author is thankful to reviewers for their careful reviews and suggestions.

Conflicts of Interest: The authors declare no conflict of interest.

\section{References}

1. Clifford, A.H.; Preston, G.B. The Algebraic Theory of Semigroups; Mathematical Surveys 7; American Mathematical Soc.: Provindence, RI, USA, 1961; Volume I, p. xv +244.

2. Rothman, N. Homomorphisms and Topological Semigroups. Ph.D. Thesis, Louisiana State University and Agricultural and Mechanical College LSU Historical Dissertations and Theses Graduate School, Baton Rouge, LA, USA, 1958.

3. Hrusak, M.; Tamariz, A.; Tkachenko, M. Pseudocompact Topological Spaces; Development in Mathematics; Springer: Morelia, Mexico, 2018; Volume 55.

4. Hofmann, K.; Mostert, P. Elements of Compact Semigroups; E. Merril Books, Inc.: Columbus, OH, USA, 1966; pp. $x$ iv +384 .

5. Numakura, K. On bicompact topological semigroups. Math. J. Okayama Univ. 1952, 1, 99-108.

6. Mukherjea, A.; Tserpes, N. A note on countably compact semigroups. J. Austral. Math. Soc. 1972, 13, 180-184. [CrossRef]

7. Robbie, D.; Svetlichny, S. An answer to A. D. Wallace's question about countably compact cancellative semigroups. Proc. Am. Math. Soc. 1996, 124, 325-330. [CrossRef]

8. Bokalo, B.; Guran, I. Sequentially compact Hausdorff cancellative semigroup is a topological group Mat. Stud. 1996, 6, 39-40.

9. Arhangelskii, A.; Tkachenko, M. Topological Groups and Related Structures; van Mill, J., Ed.; Atlantis Press: Paris, France; Amsterdam, The Netherlands, 2008.

10. Tkachenko, M. Applications of the reflection functors in paratopological groups. Topol. Appl. 2015, 192, 176-187. [CrossRef]

11. Hernández, J.; Marín, A.; Ortiz, R. Related reflections to the axioms of separation in semigroups with topologies and some applications. Res. J. Appl. Sci. 2008, 13, 675-680.

12. Tkachenko, M.; Tomita, A. Cellularity in subgroups of paratopological groups. Topol. Appl. 2015, 192, 188-197. [CrossRef]

13. Gónzalez, G. Closed Congruences on semigroups. Divulgaciones Matemáticas 2001, 9 117-121.

14. Engelking, R. General Topology; Sigma Series in Pure Mathematics; Heldermann Verlag: Berlin, Germany, 1989; Volume 6.

15. Ravsky, O. Paratopological groups II. Matematychni Studii 2002, 17, 93-101.

16. Banakh, T.; Gutik, O.; Dimitrova, S. Embedding the bicyclic semigroup into countably compact topological semigroups. Topol. Appl. 2010, 157, 2803-2814. [CrossRef]

17. Menu, J. Reflective subcategories of poset and top. In 5th Winter School on Abstract Analysis; Czechoslovak Academy of Science: Prague, Czech Republic, 1977; pp. 51-60.

18. Tkachenko, M. Axioms of separation in paratopological groups and reflection funtors. Topol. Appl. 2015, 179, 200-214. [CrossRef]

19. Tkachenko, M. Axioms of separation in semitopological groups and related functors. Topol. Appl. 2014, 161, 364-376. [CrossRef]

20. Hernández, J.; Hernández, S. Reflections in topological algebraic structures. Topol. Appl. 2020. [CrossRef] 
21. Reilly, L.; Mrsevic, M.; Vamanamurthy, M. On semiregularization topologies. Austral. Math. Soc. Ser. A 1985, 38, 40-54.

22. Reznichenko, E. Extension of functions defined on products of pseudocompact spaces and continuity of the inverse in pseudocompact groups. Topol. Appl. 1994, 9, 233-244. [CrossRef]

23. Banakh, A.R.T.T. Each paratopological group regular is completely regular. Proc. Am. Math. Soc. 2016, 145, 1373-1382. [CrossRef]

24. Martínez, J. Generalizaciones de la Compacidad en Topología General y álgebra Topológica. Master's Thesis, Universidad Autónoma de Iztapalapa, Mexico City, Mexico, 2014.

(C) 2020 by the authors. Licensee MDPI, Basel, Switzerland. This article is an open access article distributed under the terms and conditions of the Creative Commons Attribution (CC BY) license (http:/ / creativecommons.org/licenses/by/4.0/). 